\title{
LXR $\alpha$ improves myocardial glucose tolerance and reduces cardiac hypertrophy in a mouse model of obesity-induced type 2 diabetes
}

\author{
Megan V. Cannon ${ }^{1}$ - Herman H. W. Silljé ${ }^{1}$ - Jürgen W. A. Sijbesma ${ }^{2}$. \\ Mohsin A. F. Khan ${ }^{1} \cdot$ Knut R. Steffensen ${ }^{3}$ - Wiek H. van Gilst ${ }^{1} \cdot$ Rudolf A. de Boer $^{1}$
}

Received: 15 July 2015 / Accepted: 5 November 2015 / Published online: 18 December 2015

(C) The Author(s) 2015. This article is published with open access at Springerlink.com

\begin{abstract}
Aims/hypothesis Diabetic cardiomyopathy is a myocardial disease triggered by impaired insulin signalling, increased fatty acid uptake and diminished glucose utilisation. Liver X receptors (LXRs) are key transcriptional regulators of metabolic homeostasis. However, their effect in the diabetic heart is largely unknown.

Methods We cloned murine Lxr (also known as Nr1h3) behind the $\alpha$-myosin heavy chain $(\alpha M h c$; also known as $M y h \sigma)$ promoter to create transgenic $(L x r \alpha-T g)$ mice and transgenenegative littermates (wild-type [WT]). A mouse model of type 2 diabetes was induced by a high-fat diet (HFD, 60\% energy from fat) over 16 weeks and compared with a low-fat diet ( $10 \%$ energy from fat). A mouse model of type 1 diabetes was induced via streptozotocin injection over 12 weeks.

Results HFD manifested comparable increases in body weight, plasma triacylglycerol and insulin resistance per OGTT in $L x r \alpha-T g$ and WT mice. HFD significantly increased left ventricular weight by $21 \%$ in WT hearts, but only by $5 \%$
\end{abstract}

Electronic supplementary material The online version of this article (doi:10.1007/s00125-015-3827-x) contains peer-reviewed but unedited supplementary material, which is available to authorised users.

Rudolf A. de Boer

r.a.de.boer@umcg.nl

1 University of Groningen, University Medical Center Groningen, Department of Cardiology, Hanzeplein 1, 9713

GZ Groningen, the Netherlands

2 University of Groningen, University Medical Center Groningen, Department of Nuclear Medicine, Hanzeplein 1, 9713 GZ Groningen, the Netherlands

3 Division of Clinical Chemistry, Department of Laboratory Medicine, Karolinska Institutet, Karolinska University Hospital, Stockholm, Sweden in $L x r \alpha$-Tg. To elucidate metabolic effects in the heart, microPET (positron emission tomography) imaging revealed that cardiac glucose uptake was increased by 1.4 -fold in WT mice on an HFD, but further augmented by 1.7-fold in $L x r \alpha$ Tg hearts, in part through $5^{\prime}$ adenosine monophosphateactivated protein kinase (AMPK) phosphorylation and restoration of glucose transporter 4 (GLUT4). By contrast, streptozotocin-induced ablation of insulin signalling diminished cardiac glucose uptake levels and caused cardiac dysfunction, indicating that insulin may be important in LXR $\alpha$ mediated glucose uptake. Chromatin immunoprecipitation assays identified natriuretic peptides, atrial natriuretic peptide (ANP) and B-type natriuretic peptide (BNP), as potential direct targets of cardiac LXR $\alpha$ overexpression.

Conclusions/interpretation Cardiac-specific LXR $\alpha$ overexpression ameliorates the progression of HFD-induced left ventricular hypertrophy in association with increased glucose reliance and natriuretic peptide signalling during the early phase of diabetic cardiomyopathy. These findings implicate a potential protective role for LXR in targeting metabolic disturbances underlying diabetes.

Keywords Diabetic cardiomyopathy $\cdot$ Left ventricular hypertrophy $\cdot$ Liver X receptor $\cdot$ Natriuretic peptides

\begin{tabular}{|c|c|}
\hline \multicolumn{2}{|c|}{ Abbreviations } \\
\hline $\mathrm{ACC} 2$ & Acetyl-CoA carboxylase 2 \\
\hline AMPK & $\begin{array}{l}5 \text { ' Adenosine monophosphate-activated protein } \\
\text { kinase }\end{array}$ \\
\hline ANP & Atrial natriuretic peptide \\
\hline AS160 & Akt substrate of $160 \mathrm{kDa}$ \\
\hline BNP & B-type natriuretic peptide \\
\hline CAMKK $\beta$ & $\mathrm{Ca}^{2+} /$ calmodulin-dependent protein kinase \\
\hline
\end{tabular}




$\begin{array}{ll}\text { CD36 } & \text { Fatty acid translocase } \\ \text { ChIP } & \text { Chromatin immunoprecipitation } \\ \text { FA } & \text { Fatty acid } \\ {\left[{ }^{18} \text { F }\right] \text { FDG }} & \text { 2-deoxy-2-[ }{ }^{18} \text { F]fluoro-D-glucose } \\ \text { GLUT4 } & \text { Glucose transporter } 4 \\ \text { HFD } & \text { High-fat diet } \\ \text { LFD } & \text { Low-fat diet } \\ \text { LKB1 } & \text { Liver kinase B1 } \\ \text { LV } & \text { Left ventricular } \\ \text { LXR } & \text { Liver X receptor } \\ \text { LXRE } & \text { Liver X receptor response element } \\ \text { PET } & \text { Positron emission tomography } \\ \text { RXR } & \text { Retinoid X receptor } \\ \text { STZ } & \text { Streptozotocin } \\ \text { TSS } & \text { Transcriptional start site } \\ \text { WT } & \text { Wild-type }\end{array}$

Introduction

Metabolic abnormalities such as insulin resistance, disturbed glucose homeostasis, dyslipidaemia and obesity collectively predispose individuals toward the development of type 2 diabetes and are associated with an increased risk of cardiovascular disease and heart failure [1]. Moreover, obesity promotes left ventricular (LV) hypertrophy independent of hypertension [2], and LV hypertrophy is not uncommon in normotensive individuals with diabetes [3]. The underlying pathogenesis of myocardial disease induced by diabetes, referred to as diabetic cardiomyopathy, is only partially understood. However, aberrant myocardial metabolism is implicated in the early manifestation of the disease, as increased circulating fatty acids (FA) and impaired insulin signalling cause a shift in substrate usage towards exclusively favouring FA over glucose. The consequent lack of metabolic flexibility leads to lipotoxicity, impaired calcium signalling and mitochondrial dysfunction, which manifests as increased myocardial stiffness, hypertrophy and diastolic dysfunction [4].

Liver X receptors $\alpha$ and $\beta$ (LXR $\alpha$ and LXR $\beta)$ are sterolactivated transcription factors belonging to the nuclear receptor superfamily. LXRs activate target gene transcription through heterodimerization with the retinoid $\mathrm{X}$ receptor (RXR) and by interacting with an LXR response element (LXRE). LXRs have emerged as central regulators of cholesterol homeostasis and lipid and glucose metabolism, and have established anti-inflammatory and immune functions. In insulin-resistant diabetic rodents, synthetic LXR activation has been shown to reduce hyperglycaemia [5, 6] and improve peripheral insulin sensitivity $[7,8]$, effects that are mediated across multi-organ systems including suppression of gluconeogenic genes in the liver and improved peripheral glucose disposal in adipose tissue [5, 7] and skeletal muscle [9]. LXRs also play an important role in the normal and diabetic kidney through regulation of intracellular cholesterol and inflammation [10]. In the heart, activation of LXRs has been shown to attenuate pathological cardiac hypertrophy [11-13], ischaemia/reperfusion injury [14, 15], and very recently, diabetic cardiomyopathy in a $d b / d b$ mouse model of type 2 diabetes [16].

We have previously shown that mice with selective overexpression of LXR $\alpha$ in the heart ( $L x r \alpha-T g)$ demonstrate increased capacity for myocardial glucose uptake that protects against cardiac dysfunction and adverse remodelling in the adaptation to hypertrophic stress [17]. To date, the metabolic effects of LXRs in the diabetic heart have not been described. Here, we investigate the metabolic and functional consequences of cardiac LXR $\alpha$ activation in response to a metabolic challenge imposed by high-fat diet (HFD)-induced obesity and insulin resistance.

\section{Methods}

A detailed description of methods is provided in the electronic supplementary material (ESM Methods).

Generation of $\mathbf{L x} \boldsymbol{x} \boldsymbol{\alpha}$-Tg mice Transgenic mice with cardiacspecific LXR $\alpha$ overexpression were created by cloning a fulllength murine Lxr $\alpha$ (also known as Nr1h3) complementary DNA (cDNA) construct downstream of the cardiac-specific $\alpha$-myosin heavy chain ( $\alpha M h c$; also known as Myho) promoter, as previously described [17]. Mice were bred on a C57BL/ 6 background and backcrossed for six generations. Nontransgenic littermates (wild-type, WT) served as controls.

Experimental protocol Animal studies were performed in accordance with the principles of laboratory animal care (NIH publication no. 85-23, revised 1985) and with approval by the Institutional Animal Care and Use Committee of the University of Groningen, Groningen, the Netherlands. To induce a model of type 2 diabetes, diet intervention commenced in male mice of approximately 12 weeks of age; they received either an HFD (60\% energy from fat) or a nutrient-equivalent low-fat control diet (LFD; 10\% energy from fat) for 16 weeks. To induce a model of type 1 diabetes over a 12 -week period, a low-dose streptozotocin (STZ) induction protocol was performed $(50 \mathrm{mg} / \mathrm{kg} \mathrm{STZ}$ administered intraperitoneally for 5 consecutive days).

Cardiac function was assessed with echocardiography and invasive haemodynamic monitoring, and myocardial glucose uptake was determined using 2-deoxy-2-[ $\left.{ }^{18} \mathrm{~F}\right]$ fluoro-D-glucose $\left(\left[{ }^{18} \mathrm{~F}\right] \mathrm{FDG}\right)$ and microPET (positron emission tomography) imaging, as previously described $[17,18]$. An OGTT was performed whereby mice were challenged with a glucose bolus $(2 \mathrm{~g} / \mathrm{kg})$ and blood glucose levels were measured across a $3 \mathrm{~h}$ time course. LV tissue samples were used to perform 
quantitative real-time PCR and immunoblotting, biochemical assays and histological analysis, as described previously [17].

Chromatin immunoprecipitation assay Chromatin immunoprecipitation (ChIP) experiments were performed in both isolated neonatal rat ventricular myocytes and in hearts from Lxr $\alpha$-Tg and WT mice using the Pierce Agarose ChIP Kit (Thermo Scientific, Rockford, IL, USA).

Statistics Data are expressed as means \pm SEM. For group comparisons, one-way ANOVA was performed followed by Tukey's post hoc analysis. When the data were not normally distributed according to Shapiro-Wilk test for normality, Kruskal-Wallis test followed by a Mann-Whitney $U$ test for individual comparison of means were performed. A value of $p<0.05$ was considered statistically significant. Statistical analyses were performed using IBM SPSS Statistics 22 (Chicago, IL, USA).

\section{Results}

HFD induces obesity and insulin resistance in mice Prior to diet intervention, all Lxr $\alpha$-Tg mice in the LFD and HFD groups displayed comparable measures of body weight with respect to WT mice (Fig. 1a). Obesity developed similarly between Lxr $\alpha$-Tg and WT mice on HFD as both groups gained significant and proportional increases in body weight in the first 8 weeks; by 16 weeks, mice attained approximately $50 \%$ of their original body weight (Table 1 ). Mice receiving an HFD exhibited hypertriglyceridaemia and hyperinsulinaemia, as both circulating triacylglycerol (Fig. 1b) and insulin levels (Fig. 1c) were significantly elevated compared with respective LFD controls. Mice on an HFD were normoglycaemic, yet demonstrated glucose intolerance and insulin resistance per OGTT (Fig. 1d, e). AUC (Fig. 1d) was calculated from response to oral glucose challenge (Fig. 1e). In both HFD groups, post-mortem analysis revealed significant increases in liver weight of $46 \%$ and $50 \%$ for WT-HFD and Lxr $\alpha$-Tg-HFD, respectively (Table 1). These data suggest that both $L x r \alpha$-Tg and WT mice incurred comparable systemic effects from HFD intervention, resembling human insulin resistance and impaired glucose tolerance/fasting glucose. The HFD did not affect ventricular $\operatorname{LXR} \alpha$ protein expression as assessed by western blot (Fig. 1f).

Cardiac LXR $\alpha$ overexpression prevents development of LV hypertrophy induced by HFD HFD feeding over 16 weeks caused a significant increase of $21 \%$ in LV weight of WT mice, but only $5 \%$ in $L x r \alpha$-Tg mice (Fig. 2a). Expression of the adult cardiac gene, $\alpha M h c$, was significantly downregulated in WT mice on an HFD (Fig. 2b), whereas the fetal isoform, $\beta M h c$ (also known as $M y h 7$ ), was significantly higher in WT than in $L x r \alpha$-Tg mice (Fig. 2c). Transcript levels of skeletal muscle alpha-actin (Actal) were increased similarly in both HFD groups (Fig. 2d), whereas regulator of a

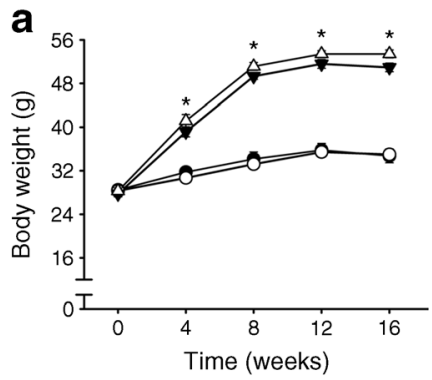

b

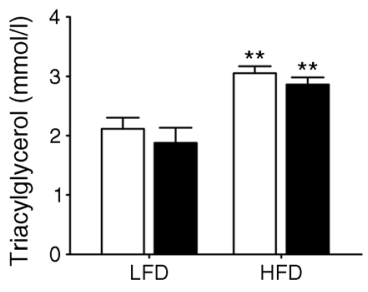

d

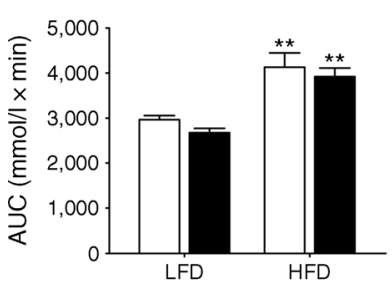

C

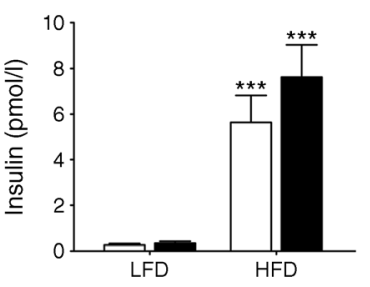

$\mathbf{f}$

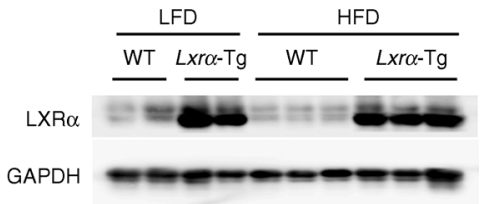

Fig. $1 L x r \alpha-T g$ and WT mice develop obesity and insulin resistance with high-fat feeding. (a, e) Circles, LFD; triangles, HFD; black symbols, WT; white symbols, $L x r \alpha$-Tg . (b-d) White bars, WT; black bars, $L x r \alpha-\mathrm{Tg}$.

(a) Body weight increased in mice after 16 weeks of an HFD ( $n=11-12 /$ group). Measurements of fasted plasma (b) triacylglycerol and (c) insulin levels ( $n=8-12$ /group). (d, e) OGTTs were performed in mice at 14 weeks

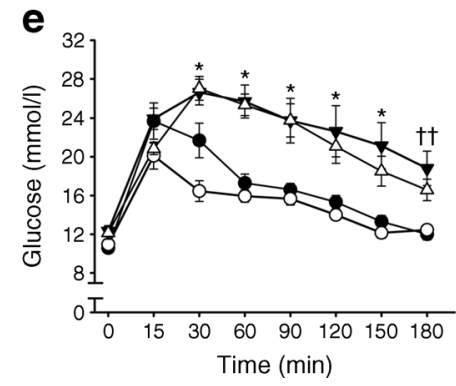

( $n=9-10$ /group). (d) AUC was calculated from (e) serial glucose measurements. All data are means \pm SEM. ${ }^{*} p<0.05$ vs respective LFD control for both $L x r \alpha-\mathrm{Tg}$ and WT; $* * p<0.01, * * * p<0.001$ vs respective LFD control; ${ }^{\dagger \dagger} p<0.01$ WT LFD vs WT HFD. (f) Western blot of LV LXR $\alpha$ protein expression in mice subjected to LFD or HFD; glyceraldehyde 3phosphate dehydrogenase (GAPDH) served as loading control 
Table 1 Biometric, echocardiographic and haemodynamic variables of $L x r \alpha$-Tg and WT mice after 16 weeks of an LFD or HFD

\begin{tabular}{|c|c|c|c|c|}
\hline \multirow[t]{2}{*}{ Variable } & \multicolumn{2}{|l|}{ LFD } & \multicolumn{2}{|l|}{ HFD } \\
\hline & WT & $\operatorname{Lxr} \alpha-\mathrm{Tg}$ & WT & $\operatorname{Lxr} \alpha-\mathrm{Tg}$ \\
\hline Post-mortem organ weight & $(n=11)$ & $(n=11)$ & $(n=12)$ & $(n=12)$ \\
\hline Body weight (g) & $34.8 \pm 1.3$ & $35.0 \pm 0.9$ & $50.9 \pm 0.8 * * *$ & $53.4 \pm 0.7 * * *$ \\
\hline LV/body weight (mg/g) & $3.5 \pm 0.1$ & $3.2 \pm 0.2$ & $2.8 \pm 0.1 * * *$ & $2.2 \pm 0.2 * * *, \dagger \dagger$ \\
\hline LV/tibia (mg/mm) & $6.9 \pm 0.1$ & $6.3 \pm 0.2$ & $8.3 \pm 0.2 * * *$ & $6.6 \pm 0.5^{*, \dagger \dagger}$ \\
\hline Kidney/tibia (mg/mm) & $23.5 \pm 0.7$ & $24.6 \pm 0.8$ & $25.1 \pm 0.6$ & $26.5 \pm 0.6$ \\
\hline Liver/tibia (mg/mm) & $92.7 \pm 5.8$ & $89.8 \pm 6.4$ & $172.8 \pm 9.1 * * *$ & $182.6 \pm 7.0 * * *$ \\
\hline Echocardiography & $(n=11)$ & $(n=10)$ & $(n=12)$ & $(n=12)$ \\
\hline Heart rate $(\mathrm{bpm})$ & $436 \pm 10$ & $436 \pm 12$ & $418 \pm 9$ & $456 \pm 13$ \\
\hline $\mathrm{LVCO} / \mathrm{BW}\left(\mathrm{ml} \mathrm{min} \mathrm{m}^{-1} \mathrm{~g}^{-1}\right)$ & $0.81 \pm 0.04$ & $0.72 \pm 0.09$ & $0.67 \pm 0.04$ & $0.70 \pm 0.04$ \\
\hline Stroke volume $(\mu \mathrm{l})$ & $66.3 \pm 2.6$ & $63.8 \pm 2.7$ & $82.9 \pm 5.0^{*}$ & $82.3 \pm 4.4 * *$ \\
\hline Fractional shortening (\%) & $40.9 \pm 1.5$ & $41.9 \pm 1.1$ & $35.2 \pm 2.2$ & $36.4 \pm 1.3$ \\
\hline LV posterior wall, diastole (mm) & $0.78 \pm 0.03$ & $0.80 \pm 0.04$ & $1.10 \pm 0.19 * *$ & $0.88 \pm 0.02 * * *$ \\
\hline LV posterior wall, systole (mm) & $1.43 \pm 0.05$ & $1.51 \pm 0.09$ & $1.45 \pm 0.06$ & $1.40 \pm 0.05$ \\
\hline $\begin{array}{l}\text { Interventricular septum, diastole } \\
(\mathrm{mm})\end{array}$ & $0.78 \pm 0.02$ & $0.72 \pm 0.01$ & $0.98 \pm 0.05 * *$ & $0.92 \pm 0.04 * * *, \dagger \dagger$ \\
\hline $\begin{array}{l}\text { Interventricular septum, systole } \\
(\mathrm{mm})\end{array}$ & $1.48 \pm 0.04$ & $1.41 \pm 0.05$ & $1.60 \pm 0.07$ & $1.56 \pm 0.06$ \\
\hline $\begin{array}{l}\text { LV internal diameter, diastole } \\
\text { (mm) }\end{array}$ & $4.00 \pm 0.10$ & $3.73 \pm 0.11$ & $4.02 \pm 0.13$ & $4.26 \pm 0.07 * *$ \\
\hline LV internal diameter, systole $(\mathrm{mm})$ & $2.37 \pm 0.10$ & $2.17 \pm 0.09$ & $2.60 \pm 0.13$ & $2.63 \pm 0.12 *$ \\
\hline E velocity $(\mathrm{m} / \mathrm{s})$ & $0.76 \pm 0.02$ & $0.71 \pm 0.02$ & $0.71 \pm 0.03$ & $0.77 \pm 0.07$ \\
\hline A velocity $(\mathrm{m} / \mathrm{s})$ & $0.54 \pm 0.02$ & $0.51 \pm 0.03$ & $0.47 \pm 0.03$ & $0.52 \pm 0.06$ \\
\hline $\mathrm{E} / \mathrm{A}$ ratio & $1.41 \pm 0.03$ & $1.43 \pm 0.06$ & $1.59 \pm 0.11$ & $1.57 \pm 0.10$ \\
\hline Deceleration time (ms) & $44.6 \pm 2.8$ & $36.7 \pm 2.5$ & $43.8 \pm 4.1$ & $37.4 \pm 2.4$ \\
\hline Haemodynamics & $(n=11)$ & $(n=11)$ & $(n=12)$ & $(n=12)$ \\
\hline \multicolumn{5}{|l|}{ Aortic pressures } \\
\hline Systolic (mmHg) & $101.1 \pm 2.2$ & $92.1 \pm 2.7$ & $105.8 \pm 2.6$ & $105.8 \pm 2.3 * *$ \\
\hline Diastolic (mmHg) & $66.5 \pm 1.9$ & $63.8 \pm 1.8$ & $69.9 \pm 2.0$ & $70.4 \pm 1.4$ \\
\hline $\begin{array}{l}\text { Mean arterial pressure } \\
(\mathrm{mmHg})\end{array}$ & $78.0 \pm 2.0$ & $73.2 \pm 2.1$ & $81.9 \pm 2.2$ & $82.2 \pm 1.6^{*}$ \\
\hline \multicolumn{5}{|l|}{ Intra-ventricular pressures } \\
\hline $\begin{array}{l}\text { LV end-systolic pressure } \\
(\mathrm{mmHg})\end{array}$ & $99.8 \pm 2.0$ & $91.5 \pm 2.7$ & $110.7 \pm 4.0$ & $103.2 \pm 2.6^{*}$ \\
\hline $\begin{array}{l}\text { LV end-diastolic pressure } \\
\qquad(\mathrm{mmHg})\end{array}$ & $7.6 \pm 2.2$ & $7.2 \pm 1.4$ & $10.4 \pm 1.2$ & $13.5 \pm 2.0$ \\
\hline $\mathrm{dP} / \mathrm{dt}_{\max }(\mathrm{mmHg})$ & $8,360 \pm 363$ & $8,420 \pm 305$ & $8,389 \pm 375$ & $8,263 \pm 293$ \\
\hline $\mathrm{dP} / \mathrm{dt}_{\min }(\mathrm{mmHg})$ & $-7,785 \pm 365$ & $-7,433 \pm 265$ & $-8,135 \pm 348$ & $-7,197 \pm 340$ \\
\hline
\end{tabular}

Data are expressed as means \pm SEM

${ }^{*} p<0.05, * * p<0.01, * * * p<0.001$, HFD versus corresponding LFD group; ${ }^{\dagger \dagger} p<0.001$ WT vs $L x r \alpha$-Tg mice bpm, beats per minute; LVCO, left ventricular cardiac output; BW, body weight calcineurin 1 (Rcan1) was significantly induced in WT but not in $L x r \alpha$-Tg mice (Fig. 2e). Interestingly, natriuretic peptides, atrial natriuretic peptide (Anp, also known as Nppa) and Btype natriuretic peptide (Bnp, also known as $N p p b$ ) were upregulated in $L x r \alpha$-Tg hearts, irrespective of diet (Fig. 2f, g).

Potential growth pathways implicated in cardiac hypertrophy and diabetic cardiomyopathy were also studied. Phosphorylated $\mathrm{Akt}^{\mathrm{Ser} 473}$ protein levels were moderately upregulated similarly between $L x r \alpha$-Tg and WT mice on HFD (ESM
Fig. 1a). In both HFD groups, downstream effects of Akt signalling strongly phosphorylated and activated the ribosomal protein S6 kinase more than threefold, with moderate effects on P70S6 kinase signalling (ESM Fig. 1b, c). HFD intervention did not result in upregulation of pathological extracellular signal-regulated kinase (ERK) and phosphorylated p44/42 mitogen-activated protein kinase (MAPK) pathways (ESM Fig. 1d). These data suggest that phospho-Akt ${ }^{\text {Ser473_S6 }}$ signalling mediates cardiac growth due to HFD in both 

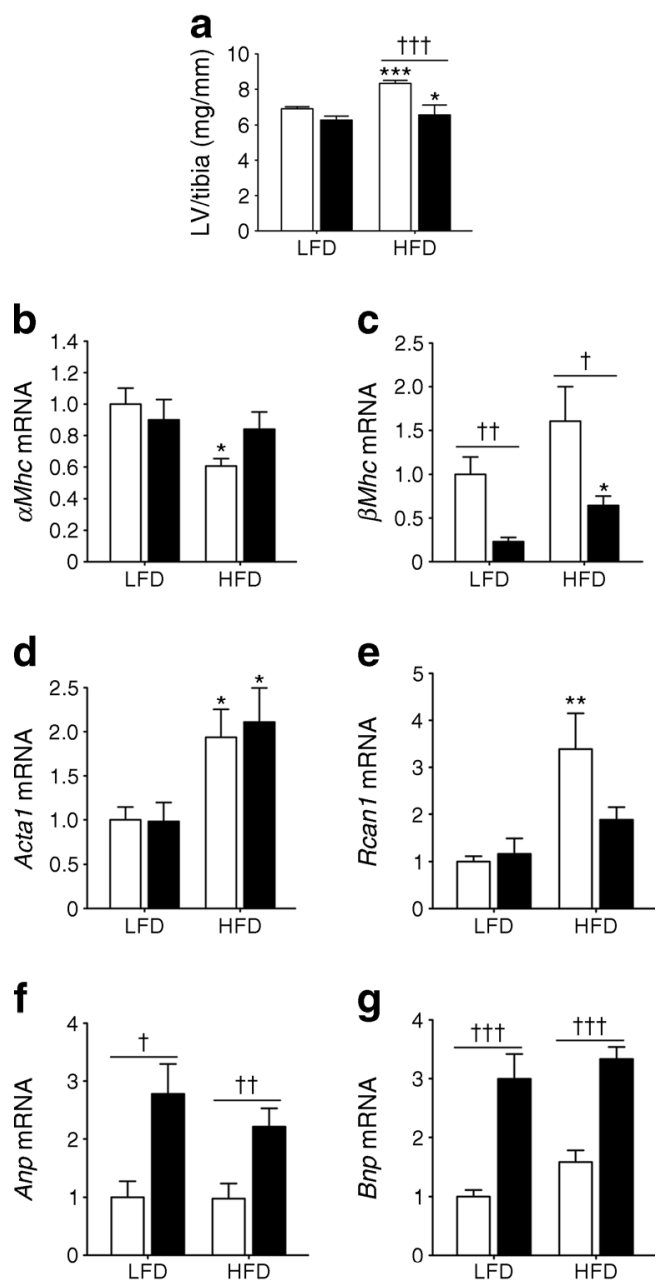

Fig. 2 Cardiac-specific LXR $\alpha$ overexpression prevents obesity-induced cardiac hypertrophy. WT (white bars), $L x r \alpha$-Tg (black bars). (a) LV to tibia length ratios in WT and $L x r \alpha$-Tg mice fed an HFD for 16 weeks; $n=$ 11-12/group. (b-g) Measurement of mRNA levels to assess hypertrophic gene expression. Values are normalised to the invariant transcript, $36 \mathrm{~b} 4$, and are expressed as fold change; $n=8-10$ /group. Data are means \pm SEM; ${ }^{*} p<0.05,{ }^{*} p<0.01,{ }^{* * *} p<0.001$ vs respective LFD control, ${ }^{\dagger} p<0.05$, ${ }^{\dagger} p<0.01,{ }^{\dagger \dagger} p<0.001$ vs WT

Lxr $\alpha$-Tg and WT mice, but is antagonised by the antihypertrophic effects of enhanced natriuretic peptides in hearts overexpressing cardiac LXR $\alpha$.

HFD causes mild hypertension and borderline diastolic and systolic dysfunction Echocardiographic and invasive haemodynamic variables of cardiac function are presented in Table 1. Mean arterial pressure and intracardiac pressures did not differ significantly between $L x r \alpha-T g$ and WT mice. Blood pressure levels tended to be lower in $L x r \alpha$-Tg mice and increased with HFD, but not in WT mice. Mice on an HFD did not display signs of diastolic dysfunction, as mitral filling velocities and deceleration time were unaltered, as well as end-diastolic LV pressure and contractility. These data indicate that the associated HFD-induced LV hypertrophy represents early structural remodelling since functional consequences are absent at this time point.

$L x r \alpha-T g$ mice demonstrate increased cardiac glucose uptake despite systemic insulin resistance We have previously demonstrated enhanced myocardial glucose uptake in $L x r \alpha-\operatorname{Tg}$ mice and in response to chronic pressure overloadinduced hypertrophy [17]. In this study, we tested the functionality of this adaptation by subjecting mice to a metabolic challenge of insulin resistance and hypertriglyceridaemia. Cardiac glucose uptake was significantly increased by 1.5 fold in $L x r \alpha-T g$ mice on an LFD compared with WT mice. HFD caused a 1.4-fold increase in glucose uptake in WT mice, but this was more markedly enhanced in $L x r \alpha-T g$ hearts (Fig. 3a, b). LV protein levels of the insulin-dependent glucose transporter 4 (GLUT4) were assessed by western blot (Fig. 3c). GLUT4 was significantly upregulated by 1.6 -fold in $L x r \alpha$-Tg mice on an LFD. However, an HFD significantly suppressed GLUT4 expression in WT mice, but this was restored by LXR $\alpha$ overexpression.

In response to an HFD, phosphorylated 5' adenosine monophosphate-activated protein kinase (AMPK) was significantly increased by 1.7 -fold in $L x r \alpha$-Tg mice compared with WT (Fig. 3d). Downstream Akt substrate of 160 kDa (AS160) phosphorylation levels were also elevated in diabetic $L x r \alpha-T g$ hearts, but not in WT-HFD hearts (Fig. 3e). Assessment of upstream kinases of AMPK revealed that liver kinase B1 (LKB1) expression was induced in $L x r \alpha-T g$ mice both at baseline and on an HFD, but not in WT mice (Fig. 3f), whereas $\mathrm{Ca}^{2+} /$ calmodulin-dependent protein kinase kinase-beta (CAMKK $\beta$ ) expression was increased by twofold in $L x r \alpha$-Tg-HFD hearts compared with WT-HFD hearts (Fig. 3g), implicating a role in $\mathrm{Ca}^{2+}$ - or contraction-stimulated glucose uptake.

Overall, these data indicate that increased basal cardiac glucose uptake in $L x r \alpha-\mathrm{Tg}$ mice is associated with induction of the insulin-dependent GLUT4 transporter, and AMPK phosphorylation may contribute to the enhanced glucose uptake levels following an HFD. Hearts overexpressing cardiac $\mathrm{LXR} \alpha$ respond to HFD intervention by upregulating LKB1 and CAMMK $\beta$, which converge to activate AMPK and promote GLUT4 translocation and upregulation via phospho-AS160.

In separate experiments, a model of type 1 diabetes was implemented (ESM Table 1, ESM Fig. 2). To further examine the effects of insulin signalling on LXR $\alpha$-mediated myocardial glucose uptake, mice were rendered insulin-deficient via STZ treatment. STZ caused a reduction of fasting insulin levels by approximately $50 \%$ (ESM Table 1 ). As a consequence, myocardial glucose uptake levels were severely depressed in both WT and $L x r \alpha-T g$ mice treated with STZ (ESM Fig. 2), which was associated with a decline in cardiac function (ESM Table 1). In $L x r \alpha$-Tg hearts subjected to STZ, the inability to upregulate phosphorylated AMPK levels was 
Fig. 3 Cardiac LXR $\alpha$ overexpression enhances myocardial glucose uptake in response to an HFD. (a, b) Mice on either an LFD or HFD underwent $\left[{ }^{18} \mathrm{~F}\right] \mathrm{FDG}$ and microPET imaging to record myocardial glucose uptake after 16 weeks on respective diets. (a) $\left[{ }^{18} \mathrm{~F}\right] \mathrm{FDG}$ uptake was determined as standard uptake value; $n=5-6$ / group. (b) Representative PET images in coronal and axial planes. (c-g) WT (white bars), Lxr $\alpha$-Tg (black bars).

Quantification of (c) GLUT4, (d)

AMPK $\alpha$ phosphorylation, (e)

AS160 phosphorylation, (f)

LKB1 and (g) CAMKK $\beta$ protein levels in LV tissue normalised to GAPDH; $n=8$ /group, except GLUT4 $n=5-6 /$ group. Data are means \pm SEM $; * p<0.05$,

$* * p<0.01, * * * p<0.001$ vs respective LFD control, ${ }^{\dagger} p<0.05$, ${ }^{\dagger} p<0.01,{ }^{\dagger \dagger} p<0.001$ vs WT
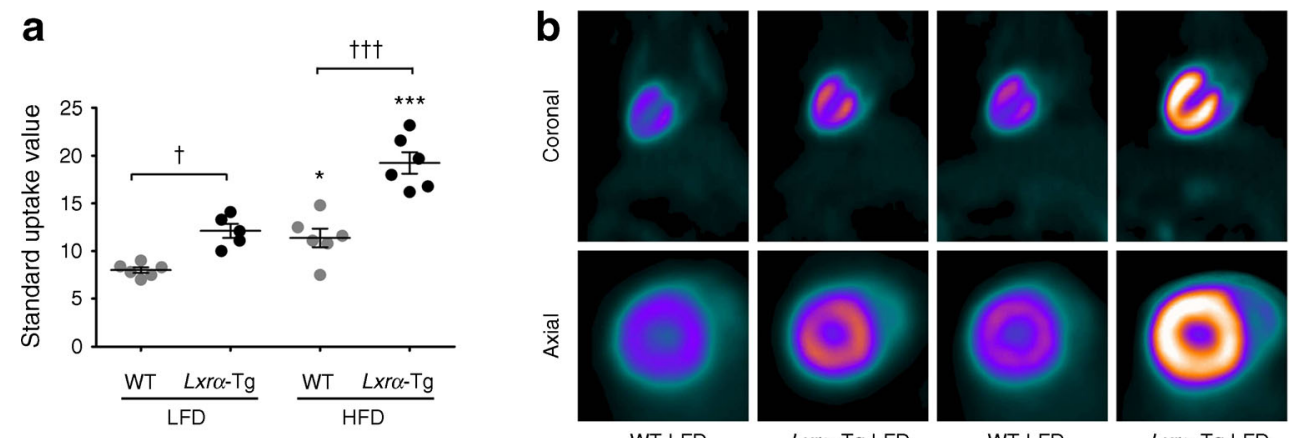

c

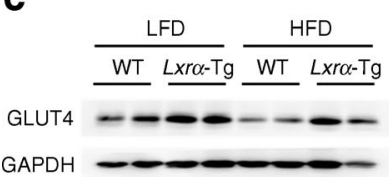

d

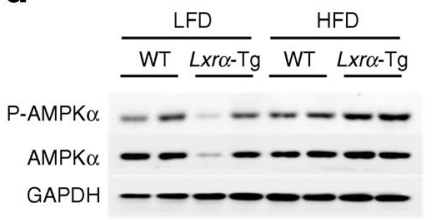

e

WT LFD

$L x r \alpha-T g L F D$
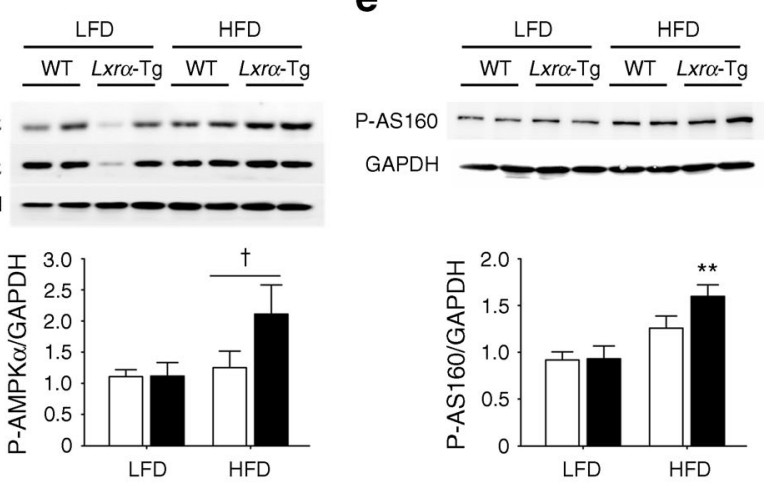

f
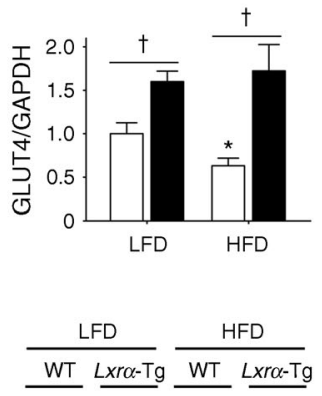

LKB1 $-=-----$
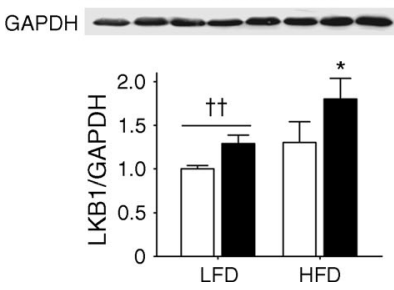

g

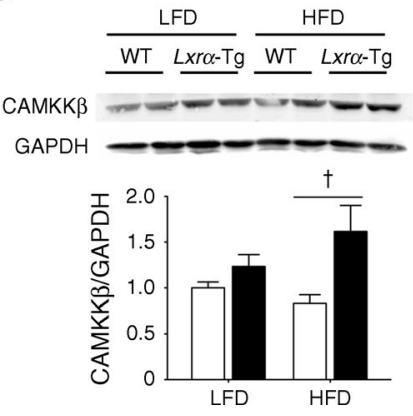

associated with significant reductions in GLUT4 expression (ESM Fig. 2c, d). Taken together, cardiac LXR $\alpha$ overexpression augments cardiac glucose uptake despite systemic insulin resistance. Nevertheless, the presence of insulin may be necessary for an LXR $\alpha$-mediated increase in glucose levels.

\section{Cardiac LXR $\alpha$ overexpression induces transcriptional} changes in lipid metabolism Genes promoting cellular and mitochondrial FA uptake such as fatty acid translocase $(C d 36$; Fig. 4a) and carnitine parmitoyltransferase I (Cptla and Cpt1b; Fig. 4c, d) were significantly downregulated in $L x r \alpha-$ $\mathrm{Tg}$ hearts in response to an HFD, suggesting a deviated shift from FA oxidation pathways. Cardiac LXR $\alpha$ negatively regulates CD36 expression and positively regulates acetyl-CoA carboxylase 2 (ACC2) expression (via an unknown mechanism; Fig. 4b). On the other hand, cardiac LXR $\alpha$ also promotes AMPK signalling (Fig. 3d), which potentially could promote FA oxidation via translocation of $\mathrm{CD} 36$ and $\mathrm{ACC} 2$ phosphorylation. HFD did not cause substantial changes in cardiac triacylglycerols, although these levels tended to be lower in $L x r \alpha$-Tg mice (ESM Fig. 3). No detectable differences in the constitutive glucose transporter 1 (Glut1; also known as Slc2a1) and hexokinase II (Hk2) mRNA levels were observed between $L x r \alpha$-Tg and WT mice (Fig. 4e, f).

Natriuretic peptides are potential direct targets of $L X R \alpha$ activation Natriuretic peptides, ANP and BNP, are induced in response to cardiac stress or injury and are antihypertrophic in their effects on the heart [19]. However, their concentrations are decreased in obesity and type 2 diabetes despite the presence of cardiac hypertrophy and dysfunction [20]. LXRs may play a regulatory role in natriuretic peptide expression since Anp and Bnp mRNA levels are significantly increased by 2.8fold and 3.0-fold, respectively, in mice with cardiac-specific LXR $\alpha$ overexpression (described previously, [17]), and 
a

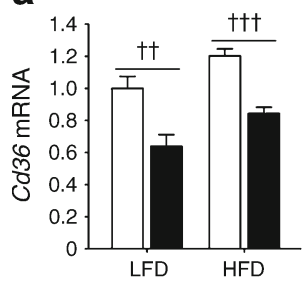

C

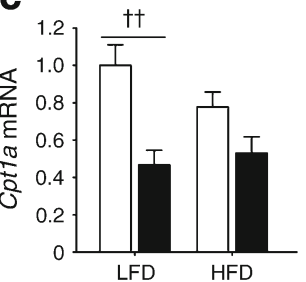

e

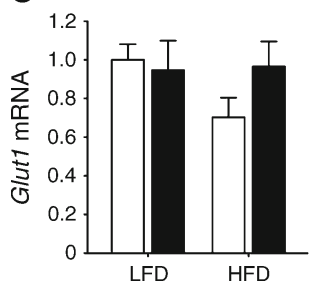

b

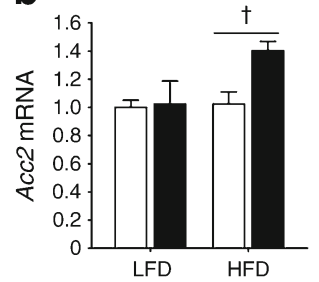

d

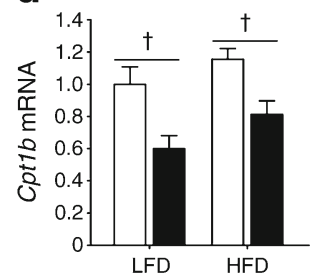

f

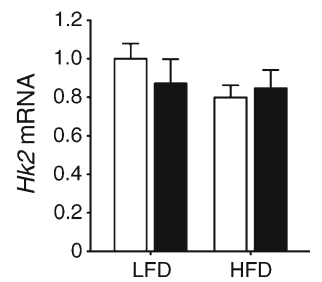

Fig. 4 Metabolic gene profile of LXR $\alpha$ transgenic hearts subjected to an HFD. (a-f) WT (white bars), $L x r \alpha$-Tg (black bars). mRNA levels were determined in LV samples following 16 weeks of either LFD or HFD for genes involved in (a) fatty acid uptake, (b-d) mitochondrial import and oxidation and $(\mathbf{e}, \mathbf{f})$ glucose uptake and metabolism. mRNA levels are normalised to $36 b 4$, and expressed as fold change; $n=8-10$ /group. Data are means \pm SEM; ${ }^{\dagger} p<0.05,{ }^{\dagger \dagger} p<0.01,{ }^{\dagger \dagger} p<0.001$ vs WT

remain upregulated in the presence of HFD-induced obesity (Fig. 2f, g).

To further evaluate the role of $\operatorname{LXR} \alpha$ on myocardial natriuretic peptide transcription, in silico analysis of the ANP/BNP region was performed using algorithms specific for the identification of LXR binding sites, or LXREs. In the mouse, several potential LXREs were identified within $\pm 50 \mathrm{~kb}$ of the Anp transcriptional start site (TSS). To determine whether the LXR/RXR heterodimer is recruited to the ANP/BNP region, ChIP analysis was performed in murine heart tissue with antibodies specific for LXR $\alpha$. An LXR $\alpha$ antibody was sufficient to pull down LXRE-containing DNA fragments of two potential LXREs, LXRE 6 and LXRE 14 (Fig. 5a-e). Agarose gel electrophoresis indicated that recruitment of $\operatorname{LXR} \alpha$ to the ANP/BNP region occurred in both WT and $L x r \alpha-T g$ mice. This gel-based assay was used for quantitative assessment of LXRE fragments by determining abundance of precipitated genomic sequences by real-time PCR. IgG antibodies served as a negative control, confirming lack of an interaction with the LXRE under investigation.

To assess whether LXRE 14 is conserved, putative binding sites for LXRE 14 in mouse, human and rat were identified using the JASPAR database (http://jaspar.genereg.net;

a

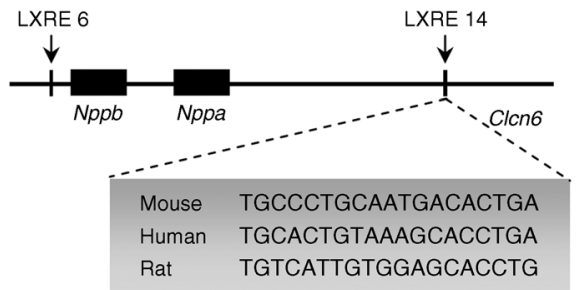

b

C
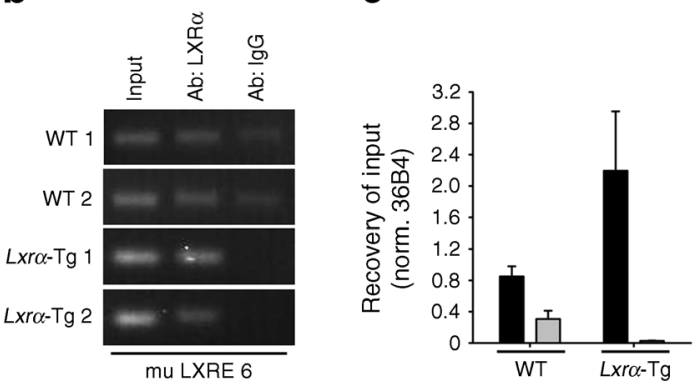

d

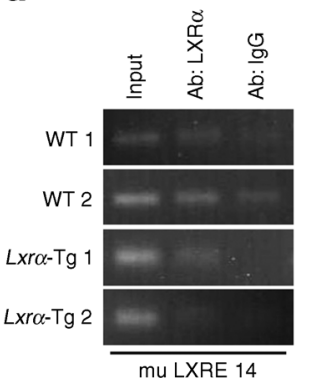

e

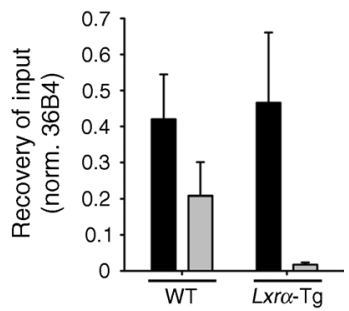

Fig. 5 Regulation of natriuretic peptide transcription by $\operatorname{LXR} \alpha$. (a) LXRE 14 binding site sequences for mouse, human and rat were identified using the JASPAR database. A region of $\pm 50 \mathrm{~kb}$ from the TSS of the Anp gene in mice was used together with syntenic regions in humans and rats. Results from this analysis revealed a highly conserved LXRE 14 binding site in the intronic region of Clcn6 in both mouse and human with a lower degree of conservation in the rat. (b-e) Chromatin immunoprecipitation assays were performed in heart samples from WT and $L x r \alpha-$ Tg mice to analyse the ANP/BNP region using antibodies directed against $\mathrm{LXR} \alpha$. Analysis of immunoprecipitated chromatin fragments are from two murine preparations per genotype, each preparation representing two pooled hearts, normalised (norm.) to 36B4. (c, e) Ab:LXR $\alpha$ (black bars), Ab:IgG (grey bars). The results are representative of the real-time PCR fragments and are analysed from gel electrophoresis. Nonspecific IgG antibodies served as a negative control. Ab, antibody; mu, murine

Fig. 5a). A region of $\pm 50 \mathrm{~kb}$ from the TSS of the mouse Anp gene was used together with syntenic regions in the human and rat. These sequences were then scanned against all available position frequency matrices using a relative profile score threshold of $80 \%$. Results from this analysis revealed an occurrence of the LXRE 14 binding site in the intronic region of Clcn6, approximately $30 \mathrm{~kb}$ downstream of the TSS of mouse Anp (score, 89\%). Comparison with orthologous regions in the human and rat identified this site as being highly conserved in human (score, $81 \%$ ). Relaxing the cut-off of the relative profile score to $70 \%$ identified 
LXRE 14 as being less stringently conserved in rat (score, $74 \%$ ). Using ChIP assay, we further validated binding of $\mathrm{LXR} \alpha$ to this site in isolated rat cardiomyocytes (ESM Fig. $4 a, b)$. LXRE 6 featured as a two-way binding site that was highly conserved in mice (score, $90 \%$ and $80 \%$ ) and rats (score, $87 \%$ and $75 \%$ ), but not in humans (ESM Fig. $4 \mathrm{c}$ ).

In conclusion, $\mathrm{LXR} \alpha$ may serve as an enhancer that binds specifically to the ANP/BNP region to regulate natriuretic peptide expression and mediate its antihypertrophic effects in the heart. Since the Anp and Bnp genes do not overlap genomically, but rather lie relatively close to each other, it is likely that functional LXREs located within this locus may influence the transcription of either gene or both.

\section{Discussion}

LXRs have been implicated as potential drug targets for the treatment of diabetes and metabolic disorders given their role in improving glucose tolerance and insulin resistance $[5,7,8$, 21]. In the present study, we investigated whether cardiac $\mathrm{LXR} \alpha$ protects the heart against diabetic cardiomyopathy. We found that cardiac-specific LXR $\alpha$ overexpression in murine hearts prevented the development of obesity-induced LV hypertrophy in the absence of overt cardiac dysfunction. Despite hyperinsulinaemia and peripheral insulin resistance, myocardial glucose uptake was remarkably enhanced in $L x r \alpha-$ Tg mice on an HFD coincident with increased AMPK activation and restoration of GLUT4.

Diabetic cardiomyopathy has become increasingly recognised as a distinct clinical entity that is characterised by the presence of cardiovascular damage in diabetic patients [3]. Although the existence of several risk factors associated with diabetes, such as hypertension and coronary artery disease, may amplify the effect of diabetes on the heart, diabetes nevertheless incurs adverse changes to the myocardium, including LV hypertrophy and fibrosis, in the absence of these confounding factors [22]. Mice with cardiac-specific LXR $\alpha$ overexpression are protected from LV hypertrophy and dysfunction following hypertrophic perturbations such as chronic pressure overload and angiotensin II stimulation [17]. Here, we extend upon these observations demonstrating that the hypertrophic response is further abrogated following a metabolic challenge of chronic hypertriglyceridaemia and hyperinsulinaemia. HFD intervention induced LV hypertrophy and molecular determinants of hypertrophic stress in WT mice in the absence of fibrosis (data not shown) and cardiac dysfunction, suggesting that hypertrophic remodelling is a very early structural manifestation of cardiomyopathic onset and progression. However, with longer duration of HFD, we speculate that obesity-induced cardiac hypertrophy may predispose mice to cardiac dysfunction, as LV hypertrophy is one of the main precursors of heart failure. Recently, the
LXR agonist GW3965 was reported to attenuate fibrosis and apoptosis and improve cardiac function in diabetic $d b / d b$ mice [16]. However, it is important to note that GW3965 also lowered body weight as well as hyperglycaemia and hypercholesterolaemia in these mice whilst improving glucose tolerance and insulin sensitivity [16], suggesting that the beneficial effects of LXR agonism in the heart are likely to be a result of less stress emanating from these systemic metabolic disturbances. In our study, these systemic variables were comparable between WT and $L x r \alpha-T g$ groups, allowing strictly for heart-specific evaluation of LXR activation.

To date, the metabolic effects of LXR in the diabetic heart have not been described. Metabolic derangements caused by HFD involve increased delivery of FA to the heart, which steers substrate preference towards exclusively FA; this adaptation, coupled with insulin resistance, limits the dependence on glucose. The heart is thus an inadvertent target of diabetes [23]. In this study, we tested the functionality of the cardiac LXR $\alpha$ transgene on myocardial glucose uptake capacity by rendering mice either insulin resistant or deficient. In response to HFD-induced insulin resistance, $L x r \alpha$-Tg mice nevertheless displayed increased myocardial glucose uptake, mediated in part by restoration of insulin-dependent GLUT4 through increased AMPK phosphorylation. By contrast, diabetes invoked by STZ-induced insulin deficiency led to impaired glucose uptake and cardiac dysfunction, indicating that cardiac LXR $\alpha$ overexpression may not be adequate to improve glucose uptake capacity in the setting of type 1 diabetes.

Interestingly, the HFD tended to mildly increase cardiac glucose uptake, which is consistent with a previous report showing that HFD-induced hyperinsulinaemia augmented glucose uptake in mice in the absence of cardiac dysfunction, and moreover, this increase in glucose flux was critical for preserving mitochondrial function [24]. Preceding the predominant utilisation of lipids that is a hallmark of diabetic cardiomyopathy, the early phase of metabolic remodelling may indeed be characterised by a heightened sensitivity to insulin that promotes glucose uptake and usage. It remains to be determined when the myocardium gradually develops insulin resistance in the face of systemic insulin resistance. Thus far, data from clinical studies are conflicting as myocardial glucose supply is reported to be either unchanged [25] or reduced [26] in diabetic patients.

Maintaining myocardial sensitivity to glucose, or reducing FA uptake may nevertheless be key in preventing the progression of diabetes-associated pathogenesis. Impaired Glut4 transcription is linked to states of insulin resistance [27]. In light of this, increasing GLUT4 levels may be cardioprotective. In this study, phosphorylation and activation of AMPK by upstream kinases, LKB1 and CAMKK $\beta$, promoted GLUT4 upregulation and translocation via AS160 phosphorylation in $L x r \alpha-T g$ hearts subjected to an HFD, but not in WT. Other studies support direct effects of LXR agonism on Glut4 transcription 
in adipose tissue and skeletal muscle, which enhance peripheral glucose clearance in rodent models of diabetes $[5,7,9]$. Moreover, expression of the FA uptake transporter gene, $C d 36$, is reciprocally downregulated in $L x r \alpha$-Tg hearts, which may confer antihypertrophic effects since $C d 36$ knockout mice are resistant to HFD-induced cardiac hypertrophy [28]. By contrast, increased CD36 expression in middle-aged mice contributes to cardiac hypertrophy, dysfunction and myocardial lipid accumulation [29].

Besides modulation of myocardial substrate metabolism, targeting of natriuretic peptides beyond their established effects on pressure-volume homeostasis may be important in preventing diabetic cardiomyopathy. A cardiometabolic link has been postulated for natriuretic peptides [20], and BNP is implicated in improved glucose utilisation through increased capillary permeability [30]. Natriuretic peptides are also known to antagonise cardiac hypertrophy independently of blood pressure [31, 32], and in humans, certain ANP- and BNP-receptor polymorphisms have been associated with LV mass in essential hypertension [33]. Both Anp and Bnp are significantly upregulated with cardiac $\mathrm{LXR} \alpha$ overexpression and their induction is unaffected by HFD-induced obesity. By contrast, natriuretic peptides are deficient in obesity and diabetes, as obese and insulin-resistant individuals display reduced circulating ANP and BNP levels [34, 35], despite the fact that these metabolic disorders increase the risk of developing cardiovascular disease and heart failure. HFD intervention did not affect natriuretic peptide expression; however, this may be model-dependant as other preclinical studies show that ANP and BNP are downregulated in diabetic $d b / d b$ mice $[36,37]$. It is currently unknown why natriuretic peptides are dysregulated in this setting. However, treatment with BNP has been shown to prevent cardiac dysfunction in $d b / d b$ mice by inhibiting cardiac hypertrophy, fibrosis and apoptosis [37], as well as the acute hypertrophic response in the diabetic rat heart [38]. LXR $\alpha$ may therefore prevent obesity-induced cardiac hypertrophy via increased local natriuretic peptide signalling. Unravelling the mechanisms by which LXR $\alpha$ modulates Anp and Bnp transcription may indeed be complex: activation of the hexosamine biosynthetic pathway through increased glucose flux leads to downstream $O$-linked glycosylation of transcriptional activators of ANP and BNP [17]. Additionally, this study identified putative LXREs in regulatory regions of the ANP/BNP promoter and ChIP analysis confirmed recruitment of $\mathrm{LXR} \alpha$ to this region, implicating natriuretic peptides as direct, heart-specific gene targets of LXR $\alpha$.

Since the structural consequences of diabetes-imposed metabolic stress on the heart are slow and progressive effects, and the clinical manifestation of symptoms is gradual, an understanding of the initial derangements is essential for early intervention. Herein, we capture a very early stage in the pathogenesis of diabetic cardiomyopathy and show that cardiac hypertrophy and glucose response to metabolic stress are preliminary developments. Promoting glucose uptake as well as natriuretic peptide signalling in the heart may be important initiatives in counteracting the progression of diabetesinduced myocardial disease. Interestingly, the recently published PARADIGM study [39] demonstrated the beneficial effects of LCZ696 in patients with heart failure. LCZ696 increases circulating BNP levels and it would be of particular interest to test this novel drug in patients with diabetes. Altogether, our results support the notion that targeting LXR $\alpha$ may be advantageous for intervening in aberrant metabolic signalling, which is a hallmark of cardiovascular disease.

Funding This study was supported by the Innovational Research Incentives Scheme program of the Netherlands Organization for Scientific Research (NWO VIDI, grant number 917.13.350).

Duality of interest The authors declare that there is no duality of interest associated with this manuscript.

Contribution statement MVC and RAdB contributed to conception and design of the study. MVC, HHWS and JWAS performed the experiments, and MVC, HHWS, JWAS, WHvG, and RAdB were involved in analysis and interpretation of the data. MAFK and KRS were responsible for conducting the bioinformatics analyses, including data acquisition and interpretation. MVC drafted the article, and HHWS, JWAS, MAFK, KRS, WHvG and RAdB revised it critically for important intellectual content. All authors have approved the final version of the manuscript. MVC is responsible for the integrity of the work as a whole.

Open Access This article is distributed under the terms of the Creative Commons Attribution 4.0 International License (http:// creativecommons.org/licenses/by/4.0/), which permits unrestricted use, distribution, and reproduction in any medium, provided you give appropriate credit to the original author(s) and the source, provide a link to the Creative Commons license, and indicate if changes were made.

\section{References}

1. Ingelsson E, Sundstrom J, Arnlov J, Zethelius B, Lind L (2005) Insulin resistance and risk of congestive heart failure. JAMA 294: 334-341

2. Woodiwiss AJ, Libhaber CD, Majane OH, Libhaber E, Maseko M, Norton GR (2008) Obesity promotes left ventricular concentric rather than eccentric geometric remodeling and hypertrophy independent of blood pressure. Am J Hypertens 21:1144-1151

3. Galderisi M, Anderson KM, Wilson PW, Levy D (1991) Echocardiographic evidence for the existence of a distinct diabetic cardiomyopathy (the Framingham Heart Study). Am J Cardiol 68: 85-89

4. Taegtmeyer H, McNulty P, Young ME (2002) Adaptation and maladaptation of the heart in diabetes: part I: general concepts. Circulation 105:1727-1733

5. Cao G, Liang Y, Broderick CL et al (2003) Antidiabetic action of a liver $\mathrm{x}$ receptor agonist mediated by inhibition of hepatic gluconeogenesis. J Biol Chem 278:1131-1136

6. Liu Y, Yan C, Wang Y et al (2006) Liver X receptor agonist T0901317 inhibition of glucocorticoid receptor expression in 
hepatocytes may contribute to the amelioration of diabetic syndrome in $\mathrm{db} / \mathrm{db}$ mice. Endocrinology 147:5061-5068

7. Laffitte BA, Chao LC, Li J et al (2003) Activation of liver X receptor improves glucose tolerance through coordinate regulation of glucose metabolism in liver and adipose tissue. Proc Natl Acad Sci U S A 100:5419-5424

8. Grefhorst A, van Dijk TH, Hammer A et al (2005) Differential effects of pharmacological liver $\mathrm{X}$ receptor activation on hepatic and peripheral insulin sensitivity in lean and ob/ob mice. Am J Physiol Endocrinol Metab 289:E829-E838

9. Baranowski M, Zabielski P, Blachnio-Zabielska AU, Harasim E, Chabowski A, Gorski J (2014) Insulin-sensitizing effect of LXR agonist T0901317 in high-fat fed rats is associated with restored muscle GLUT4 expression and insulin-stimulated AS160 phosphorylation. Cell Physiol Biochem 33:1047-1057

10. Patel M, Wang XX, Magomedova L et al (2014) Liver X receptors preserve renal glomerular integrity under normoglycaemia and in diabetes in mice. Diabetologia 57:435-446

11. Wu S, Yin R, Ernest R et al (2009) Liver X receptors are negative regulators of cardiac hypertrophy via suppressing NF-kappaB signalling. Cardiovasc Res 84:119-126

12. Kuipers I, Li J, Vreeswijk-Baudoin I et al (2010) Activation of liver $\mathrm{X}$ receptors with T0901317 attenuates cardiac hypertrophy in vivo. Eur J Heart Fail 12:1042-1050

13. Cannon MV, Yu H, Candido WM et al (2015) The liver X receptor agonist AZ876 protects against pathological cardiac hypertrophy and fibrosis without lipogenic side effects. Eur J Heart Fail 17: 273-282

14. Lei P, Baysa A, Nebb HI et al (2013) Activation of liver X receptors in the heart leads to accumulation of intracellular lipids and attenuation of ischemia-reperfusion injury. Basic Res Cardiol 108:323

15. He Q, Pu J, Yuan A et al (2014) Activation of liver-X-receptor alpha but not liver-X-receptor beta protects against myocardial ischemia/ reperfusion injury. Circ Heart Fail 7:1032-1041

16. $\mathrm{He} \mathrm{Q}, \mathrm{Pu}$ J, Yuan A et al (2014) Liver X receptor agonist treatment attenuates cardiac dysfunction in type 2 diabetic $\mathrm{db} / \mathrm{db}$ mice. Cardiovasc Diabetol 13:149

17. Cannon MV, Sillje HH, Sijbesma JW et al (2015) Cardiac LXRalpha protects against pathological cardiac hypertrophy and dysfunction by enhancing glucose uptake and utilization. EMBO Mol Med 7:1229-1243

18. Yu L, Ruifrok WP, Meissner M et al (2013) Genetic and pharmacological inhibition of galectin-3 prevents cardiac remodeling by interfering with myocardial fibrogenesis. Circ Heart Fail 6:107-117

19. Nishikimi T, Maeda N, Matsuoka H (2006) The role of natriuretic peptides in cardioprotection. Cardiovasc Res 69:318-328

20. Zois NE, Bartels ED, Hunter I, Kousholt BS, Olsen LH, Goetze JP (2014) Natriuretic peptides in cardiometabolic regulation and disease. Nat Rev Cardiol 11:403-412

21. Commerford SR, Vargas L, Dorfman SE et al (2007) Dissection of the insulin-sensitizing effect of liver $\mathrm{X}$ receptor ligands. Mol Endocrinol 21:3002-3012

22. Rubler S, Dlugash J, Yuceoglu YZ, Kumral T, Branwood AW, Grishman A (1972) New type of cardiomyopathy associated with diabetic glomerulosclerosis. Am J Cardiol 30:595-602

23. Ouwens DM, Boer C, Fodor M et al (2005) Cardiac dysfunction induced by high-fat diet is associated with altered myocardial insulin signalling in rats. Diabetologia 48:1229-1237
24. Gupte AA, Minze LJ, Reyes M et al (2013) High-fat feeding-induced hyperinsulinemia increases cardiac glucose uptake and mitochondrial function despite peripheral insulin resistance. Endocrinology 154:2650-2662

25. Ohtake T, Yokoyama I, Watanabe T et al (1995) Myocardial glucose metabolism in noninsulin-dependent diabetes mellitus patients evaluated by FDG-PET. J Nucl Med 36:456-463

26. Iozzo P, Chareonthaitawee P, Dutka D, Betteridge DJ, Ferrannini E, Camici PG (2002) Independent association of type 2 diabetes and coronary artery disease with myocardial insulin resistance. Diabetes 51:3020-3024

27. Griesel BA, Weems J, Russell RA, Abel ED, Humphries K, Olson AL (2010) Acute inhibition of fatty acid import inhibits GLUT4 transcription in adipose tissue, but not skeletal or cardiac muscle tissue, partly through liver X receptor (LXR) signaling. Diabetes 59:800-807

28. Sung MM, Koonen DP, Soltys CL, Jacobs RL, Febbraio M, Dyck JR (2011) Increased CD36 expression in middle-aged mice contributes to obesity-related cardiac hypertrophy in the absence of cardiac dysfunction. J Mol Med (Berl) 89:459-469

29. Koonen DP, Febbraio M, Bonnet S et al (2007) CD36 expression contributes to age-induced cardiomyopathy in mice. Circulation $116: 2139-2147$

30. Welsh P, McMurray JJ (2012) B-type natriuretic peptide and glycaemia: an emerging cardiometabolic pathway? Diabetologia 55:1240-1243

31. Holtwick R, van Eickels M, Skryabin BV et al (2003) Pressureindependent cardiac hypertrophy in mice with cardiomyocyterestricted inactivation of the atrial natriuretic peptide receptor guanylyl cyclase-A. J Clin Invest 111:1399-1407

32. Franco V, Chen YF, Oparil S et al (2004) Atrial natriuretic peptide dose-dependently inhibits pressure overload-induced cardiac remodeling. Hypertension 44:746-750

33. Rubattu S, Bigatti G, Evangelista A et al (2006) Association of atrial natriuretic peptide and type a natriuretic peptide receptor gene polymorphisms with left ventricular mass in human essential hypertension. J Am Coll Cardiol 48:499-505

34. Wang TJ, Larson MG, Levy D et al (2004) Impact of obesity on plasma natriuretic peptide levels. Circulation 109:594-600

35. Olsen MH, Hansen TW, Christensen MK et al (2005) N-terminal pro brain natriuretic peptide is inversely related to metabolic cardiovascular risk factors and the metabolic syndrome. Hypertension 46:660-666

36. Gutkowska J, Broderick TL, Bogdan D, Wang D, Lavoie JM, Jankowski M (2009) Downregulation of oxytocin and natriuretic peptides in diabetes: possible implications in cardiomyopathy. J Physiol 587:4725-4736

37. Plante E, Menaouar A, Danalache BA, Broderick TL, Jankowski M, Gutkowska J (2014) Treatment with brain natriuretic peptide prevents the development of cardiac dysfunction in obese diabetic $\mathrm{db} / \mathrm{db}$ mice. Diabetologia 57:1257-1267

38. Rosenkranz AC, Hood SG, Woods RL, Dusting GJ, Ritchie RH (2003) B-type natriuretic peptide prevents acute hypertrophic responses in the diabetic rat heart: importance of cyclic GMP. Diabetes 52:2389-2395

39. McMurray JJ, Packer M, Desai AS et al (2014) Angiotensinneprilysin inhibition versus enalapril in heart failure. $\mathrm{N}$ Engl $\mathrm{J}$ Med 371:993-1004 